\title{
THE LARGE-SCALE VARIATIONS OF QUASI-STELLAR OBJECTS
}

\author{
W. H. MCCREA \\ University of Sussex, Falmer, Great Britain
}

The proposition for consideration is:

A QSO is an approximately standard object: it has an approximately standard history consisting mainly of an increase of optical brightness to a very high peak, and a subsequent decline. I suggest that the history has a relevant duration of about $10^{6} \mathrm{yr}$, and that the total increase of brightness is by a factor about $10^{3}$, while the mean brightness is of the order of $1 \%$ of the peak brightness (so that the peak is rather sharp).

The following corollaries would result:

(1) The energy requirement of an individual QSO would be less than that usually assumed (a few percent);

(2) The spread of intrinsic brightness of QSOs would be due mainly to the variation;

(3) QSOs would have a particular greatest intrinsic brightness, the same for all distances;

(4) Since, by hypothesis, the peak brightness is very high, it is plausible that QSOs with very large redshift be detectable. This need not demand some QSOs of exceptionally great energy;

(5) The variation would tend to obscure the effect of distance upon apparent magnitude, and so to mask the Hubble relation, but we might get a significant relation from the brightest QSOs at each redshift.

(6) Most optical properties would be statistically the same for all QSOs at the same stage in their history. If QSOs at redshifts $z_{1}$ and $z_{2}$ show any similar variabilities to which we assign characteristic times $T_{1}$ and $T_{2}$, then we should expect to find

$$
T_{1} / T_{2}=\left(z_{1}+1\right) /\left(z_{2}+1\right)
$$

(7) Since the calculation of intrinsic properties from the observations is modeldependent, then, conversely, we could seek a model that gives the same intrinsic luminosity-function for all values of the redshift, $z$.

Equation (1) would provide a powerful test of the cosmological, or at any rate the Doppler, character of $z$. For if the line-shift were, say, gravitational, it is unlikely that variations in the continuous emission would be subject to the same 'shift'. The test could be applied in a quite crude fashion. We could take all the light curves, such as those discussed by Harlan J. Smith and by T. D. Kinman, and plot those for QSOs having, say, $z>1$, on half the time scale for those having $z<1$ and see if the two lots are more similar than when they are plotted on the same time scale.

The corollaries 5, 6 and 7 may provide ways of exploiting the large $z$-values of QSOs 
for cosmological tests: hitherto not much success has been achieved in this respect.

\section{Evidence in Support of the Proposition}

It seems to be generally agreed that a QSO has an active life of the order of $10^{6} \mathrm{yr}$. So QSOs must be continually appearing and disappearing, i.e. rising to the typical QSO brightness and then fading. It is economy of hypothesis to accept this as the main reason for the great spread of intrinsic luminosities.

On the $q_{0}=+1$ Friedman model, the intrinsic brightness of the brightest QSOs is indeed nearly constant. In particular, I find that the object $4 \mathrm{C} 05.34$ recently found by C. R. Lynds and Derek Wills to have $z=2.877$ has almost exactly the same absolute luminosity as 3C 273 on this model.

There are the well-known cases of 3C 446 and 5C 2.56 (recently discovered by E. M. Burbidge) that show great variations in brightness so that at any rate some of the spread in brightness must be due to variations.

It has always seemed significant that the energy required by a QSO was calculated to be not a great deal more than what could be produced by known energy-sources. If by allowing for variations we find that the energy requirement is appreciably reduced, the fact that the requirement can then be met by known sources appears to be an argument in favour of such behavior.

\section{Remarks}

There is nothing basically new in these ideas. The discussion about density evolution vs luminosity evolution is essentially the same. However, it seems opportune to call attention to some of the foregoing considerations.

The suggestions made here are simple and tentative, but some of the consequences would follow even were the main proposition only very approximately valid. Also there may be complicating factors, e.g. a QSO may have several main peaks of brightness, and there might be directional properties.

The radio properties of a QSO are probably secondary consequences of the main mechanism, and so these properties may not be accommodated in a simple discussion. One would be inclined to suppose that the radio properties may be associated more with the later part of the life of a QSO.

\section{Discussion}

Mackay: It may be necessary to consider QSO lifetimes considerably in excess of $10^{6} \mathrm{yr}$. There are now data on a number of QSOs whose radio components are separated by great distances from the optical object and which appear to have ages of the order of $10^{6} \mathrm{yr}$. However, in these cases the QSO is still optically bright - perhaps only one magnitude fainter than the brightest QSOs - and in some of these sources another component is observed coincident with the optical QSO.

McCrea: As I have mentioned in the written account, I should suppose that there may be more than one outburst in certain QSOs. Consequently, in the case mentioned by Dr Mackay the extended features may be associated with a much earlier outburst. 